\title{
The influence of adaptation and stochastic fluctuations on spontaneous perceptual changes for bistable stimuli
}

\author{
HOWARD S. HOCK \\ Florida Atlantic University, Boca Raton, Florida \\ GREGOR SCHÖNER \\ Laboratoire de Neurosciences Cognitives, Marseille, France \\ and \\ AUDREY VOSS \\ Florida Atlantic University, Boca Raton, Florida
}

\begin{abstract}
Spontaneous perceptual change was studied by measuring the probabilities of the first two spontaneous pattern switches as a function of time following the onset of a bistable apparent quartet for which either horizontal or vertical motion is perceived. Contrary to the classical satiation hypothesis (Köhler \& Wallach, 1944), differential time-dependent adaptation of the perceived compared with the unperceived motion directions was not necessary to account for the first spontaneous switch. In addition, adaptation of the perceived motion accompanied by recovery from adaptation of the unperceived motion was not necessary to account for the increased probability of the second spontaneous switch. It was concluded that regardless of possible adaptation effects, stochastic fluctuations are necessary for the actual reversal of activation levels that produces the spontaneous switch. When the difference in detector activation is reduced by differential adaptation of competing motion detectors (or by the occurrence of a prior spontaneous pattern change), smaller stochastic fluctuations are sufficient to reverse the relative activation of competing detectors. Thus, adaptation can increase the probability of spontaneous switches without directly causing them.
\end{abstract}

The perception of reversible figures has been studied since the earliest days of experimental psychology (Jastrow, 1900; McDougall, 1906). There are two closely related aspects. The first is that reversible figures are multistable in the sense that the same stimulus can result in the perception of at least two qualitatively different patterns. The second is that the percept can spontaneously change from one pattern to another; the change is spontaneous in the sense that it does not depend on changes in the stimulus.

The classical explanation proposes that satiation, or neural fatigue, is responsible for spontaneous perceptual change (Köhler \& Wallach, 1944). Since some passage of time is required prior to the occurrence of a spontaneous change, the satiation hypothesis specifies that an initially established percept loses "strength" (activation) as a function of time, the time course being slow relative to the time required for the formation of the percept (otherwise nothing would ever be perceived). Thus, spontaneous perceptual change occurs when the activation of detecting units that are the basis for a percept are reduced so much that

Correspondence should be addressed to H. S. Hock, Department of Psychology, Florida Atlantic University, Boca Raton, FL 33431 (email: hockhs@fau.edu). it falls below the activation of detecting units that are the basis for the competing percept.

It has long been known that prolonged exposure can result in adaptive reductions in threshold-level perceptual sensitivity - see, for example, Blakemore and Campbell (1969) for contrast sensitivity to static sine gratings, and Pantle and Sekuler $(1968,1969)$ and Sekuler and Ganz (1963) for contrast sensitivity to drifting gratings. At suprathreshold levels, adaptive changes in sensitivity reduce perceived contrast (Troxler, 1804) and slow perceived motion (Thompson, 1981). Thus, the loss of activation specified by the satiation hypothesis can be attributed to adaptive reductions in the sensitivity of the detecting units that are activated by some or all of the features of the bistable stimulus.

However, what is central to the satiation hypothesis is not the occurrence of adaptation, but that adaptation is sufficient for the reversal of the relative activation of detecting units for the competing patterns. It is the reversal in relative activation that results in spontaneous change in the pattern that is perceived. Thus, the satiation hypothesis requires that spontaneous perceptual switching occur only when there are differential changes in the sensitivity of detectors associated with the perceived compared with the unperceived pattern. 
Often cited as support for the satiation hypothesis is evidence that the time between successive switches for a continuously examined bistable stimulus gradually decreases with each succeeding switch, eventually asymptoting at a relatively constant interswitch duration after 30-75 sec (see, e.g., Babich \& Standing, 1981; Cohen, 1959). The typical explanation for the increasing switching rate is that over a series of switches, patterns that had been perceived beforehand are reinstated in perception even though they have not returned to full "strength" (Cornwell, 1976; Spitz \& Lipman, 1962). To make this concrete, let us call the initially perceived pattern for a bistable stimulus "Pattern A." After some period of adaptive reduction in the activation of detectors that are the basis for Pattern A, it is replaced in perception by Pattern B. While Pattern B is being perceived (and losing its activation as a result of adaptive reductions in the sensitivity of detectors that are the basis for its formation), the sensitivity of detectors that are the basis for Pattern A recover. If the recovery rate is slower than the adaptation rate, Pattern $A$ will be reestablished in perception when its activation exceeds that of Pattern B, but it will have been restored too soon for its activation to return to its initial level. Hence, it will adapt to the "switching point" sooner for the second switch than it did for the first one. Accordingly, the time between switches between the two patterns will decrease on successive switches.

Thus, explanations of experiments demonstrating decreases in the time between successive switches make a further claim regarding differential changes in the sensitivity of detectors associated with the perceived compared with the unperceived pattern. That is, over time the sensitivity of detectors that respond to features of the perceived pattern decreases while the sensitivity of detectors that respond to features of the currently unperceived, previously adapted pattern increases. Once again, it is assumed that a perceptual switch occurs when the relative activation of detectors associated with the two patterns reverses as a result of this differential adaptation/recovery.

Also relevant to the satiation hypothesis are experiments in which prior adaptation influences the perception of bistable stimuli. Prior adaptation in these experiments involves a period of time viewing a version of a bistable stimulus that is strongly biased toward one of two alternative percepts. This presumably reduces the sensitivity of the detecting units on which the adapting percept is based, so when the biased version is replaced by a bistable (neutral) version of the stimulus, the effect of the prior adaptation is to increase the likelihood of perceiving the alternative pattern (Carlson, 1953; Hochberg, 1950; Kruse, Stadler, \& Wehner, 1986; Long, Toppino, \& Mondin, 1992; Nawrot \& Blake, 1989).

In Kruse et al.'s (1986) experiments, apparent motion stimuli were formed by simultaneously presenting two points of light corresponding to the diagonally opposite corners of an imaginary rectangle, then simultaneously presenting the two points of lights corresponding to the other diagonally opposite corners, then the first pair again, then the second pair again, and so on. Both dots are per- ceived to move vertically, though in opposite directions, or both dots are perceived to move horizontally, again in opposite directions (Figure 1). Kruse et al.'s adapting quartet had an aspect ratio (vertical/horizontal distance between the dot positions) of 2.26 , which strongly favors the perception of horizontal motion. The effect of prolonged viewing of the horizontally biased adapting quartet was to increase the likelihood of vertical motion being perceived for the subsequently presented neutral test quartet (its aspect ratio was 1.13 ), presumably because of the adaptation of the horizontal motion perceived during the adaptation interval.

In a recent study based on Kruse et al.'s (1986) paradigm, Hock, Schöner, and Hochstein (1996) determined that adaptation effects for the motion quartet are not necessarily restricted to the motion direction perceived during adaptation; that is, if horizontal motion is perceived during the adaptation phase, effects of adaptation can be observed during the test phase for vertical as well as horizontal motion. A critical aspect of Hock et al.'s experiments was that the analysis was restricted to trials for which horizontal motion was perceived for the entire adaptation phase. Trials for which vertical motion was perceived anytime during the adaptation phase were not analyzed. Hock et al. found that vertical motion was perceived more often during the test phase when the horizontal motion perceived throughout the adaptation phase was stable (unlikely to spontaneously change to vertical motion) compared with when it was relatively unstable. They concluded that when the adapting horizontal motion was stable, reductions in sensitivity occurred predominantly for the motion directions perceived during adaptation (i.e., there was differential adaptation of the perceived and unperceived motion directions). However, when the adapting motion was relatively unstable, reductions in sensitivity occurred both for the horizontal motion directions that were perceived and for the vertical motion directions that were not perceived during the adaptation phase.

In a further experimental test of this conclusion, Hock et al.'s (1996) adapting stimulus was either a horizontally oriented trapezoidal motion quartet (its parallel sides were horizontal) or a $90^{\circ}$ rotated, vertically oriented trapezoidal motion quartet (its parallel sides were vertical). The test stimulus was a neutral rectangular quartet. When motion was perceived along the oblique sides of the trapezoidal quartets for the entire adaptation phase, more vertical motion was perceived for the rectangular test quartet when the adapting trapezoidal quartet had been horizontal than when the adapting trapezoidal quartet had been vertical. This provided evidence for adaptation of unperceived (but possible) horizontal motion by the horizontally oriented trapezoidal quartet and adaptation of unperceived (but possible) vertical motion by the vertically oriented trapezoidal quartet.

Hock et al.'s (1996) evidence for adaptation affecting both the perceived and unperceived motion directions of motion quartets directly challenges the central assumption of the satiation hypothesis: that all adaptation (sati- 
ation) effects are associated with detecting units that are the basis for the perceived pattern, and that detecting units associated with the alternative pattern are either not adapting or are recovering from the effects of prior adaptation. A further implication of Hock et al.'s results is that if detecting units associated with both the perceived and unperceived pattern adapt, the time course of their adaptation might be similar. One of the possible percepts therefore need not gain an "advantage" relative to the other as a result of adaptation. Thus, even if adaptation effects are time dependent, the occurrence of spontaneous perceptual changes need not be time dependent if adaptation proceeds at the same rate for the perceived and unperceived patterns.

\section{THE PRESENT STUDY}

In Experiment 1, the first spontaneous change in perceived motion direction during each trial was studied for motion quartets with different aspect ratios. The objective was to determine whether at least one condition could be found for which temporally independent spontaneous switching could be reliably observed. This would provide evidence that spontaneous switching can occur without differential adaptation-that is, without greater adaptation of perceived than of unperceived motion directions. Temporally independent spontaneous switching would indicate either similar adaptation occurring over the same time course for the perceived and unperceived motion directions, or no adaptation for either. Stochastic fluctuations in relative activation would then be indicated as necessary for spontaneous perceptual change (although under other conditions, differential adaptation might influence the size, and therefore the probability of the stochastic fluctuation required to produce a spontaneous switch).

Experiment 2 addressed the evidence for the increase in switching probability with successive switches (i.e., decreases in the time between switches) obtained in earlier studies. More specifically, it was concerned with explanations that attribute this evidence to differential changes in sensitivity for detectors that respond to features of the perceived and unperceived patterns. That is, detectors for perceived features adapt (decrease in sensitivity), whereas detectors for unperceived features recover from earlier adaptation (increase in sensitivity). The objective of Experiment 2 was to verify previous evidence that the probability of the second switch is greater than that of the first, but under conditions in which there is no preceding differential adaptation of the perceived and unperceived motion directions. If there is recovery from adaptation, it would not account for the increase in switch rate because recovery would be concurrent for horizontal and vertical motion detectors that have adapted concurrently. Again, stochastic fluctuations in relative activation might account for the results. For example, stochastic fluctuations that overcome an initial advantage in activation for horizontal motion (the first switch) might result, on average, in a relatively small activational ad- vantage for the newly perceived vertical motion. As a result, a stochastic fluctuation that again reverses the relative activation of vertical and horizontal motion detectors (the second switch) is more likely because the size of the required stochastic fluctuation would be smaller.

\section{EXPERIMENT 1}

As indicated above, the bistable stimuli studied in this article were motion quartets. These stimuli were originally studied by von Schiller (1933); Metzger (1941/1975); and Hoeth (1968); and more recently by Ramachandran and Anstis (1985); Kruse et al. (1986); Hock, Kelso, and Schöner (1993); and He and Nakayama (1994). They are composed of small visual elements (usually dots) presented in the corners of an imaginary rectangle in such a way that smooth apparent motion is seen in either vertical or horizontal directions. Although the simultaneous perception of horizontal and vertical motion is logically possible for these stimuli, either horizontal motion (Figure la) or vertical motion (Figure $1 \mathrm{~b}$ ) is perceived, but never both at the same time. Of interest was whether the first spontaneous change between these two percepts increased or remained constant as a function of time.

Critical to any experimental study of spontaneous pattern change is the time scale of adaptation and stochastic fluctuations in relation to the time scale of the experimental trials. That is, trial duration must be of the same order of magnitude as the time course of adaptation, and although stochastic fluctuations can be virtually instantaneous, experimental trials must be of sufficient duration to capture the relatively infrequent stochastic fluctuations

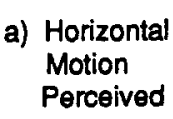

\section{Motion
Perceived}

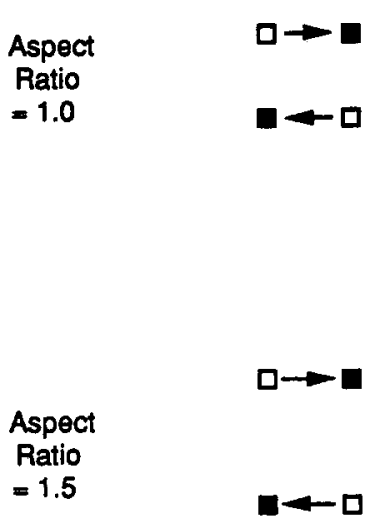

b) Vertical Motion Perceived

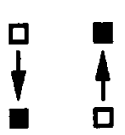

Figure 1. Illustration of the horizontal (a) and vertical (b) motion patterns perceived for apparent motion quartets with aspect ratios of 1.0 and 1.5 (the vertical divided by the horizontal distance between dot positions). The unfilled and filled squares indicate the dot positions on odd- and even-numbered frames, respectively (the dot positions denoted by the unfilled squares precede the dot positions denoted by the filled squares). 
that are large enough to produce spontaneous perceptual changes. Furthermore, measuring the time course of spontaneous pattern change for even the first change poses a significant experimental problem. When subjects are instructed to respond as soon as they have experienced a pattern change, it is impossible to accurately establish when the switch took place by extrapolating backward in time and factoring out the motor and decision aspects of their response. Hock et al. (1993) have eliminated this problem with an experimental technique for measuring the time course of perceptual change without measuring response time. The technique involves varying the duration of each trial and having subjects wait until the end of the trial before reporting (without speed stress) whether or not there was a change in the perceived pattern anytime during the trial. Brief trials provide a "temporal window" for only the quickest switches; longer duration trials provide a "wider" temporal window that can capture switches that occur after longer temporal intervals.

Subjects' responses indicated the frequency with which they perceived at least one perceptual switch during the course of a trial, but our interest was in the probability of a switch per unit of time. Does it change over time, or is the probability of a switch temporally independent? On the basis of the assumption of temporal independence (i.e., the probability of a switch per unit of time remains constant), we calculated switching functions for the probability of " 1 or more" switches as a function of trial duration and compared them with empirically obtained "l or more" switching functions. If spontaneous perceptual changes occur only as a result of differential, timedependent adaptation of the perceived compared with the unperceived motion directions (as required by the satiation hypothesis), the probability of at least one perceptual change would never follow a calculated, temporalindependence switching function.

\section{Method}

Subjects. The 3 subjects ( 1 was Author A.V.) were all research assistants in the visual perception laboratory at Florida Atlantic University. All had normal or corrected-to-normal vision, and all were well practiced.

Stimuli. Pairs of white dots were presented over a series of frames against the dark background of a Macintosh II RGB monitor. The size of each dot was 1 pixel, intercepting a visual angle of $1.6^{\prime}\left(0.027^{\circ}\right)$ from the $80-\mathrm{cm}$ viewing distance (maintained by a head restraint). The luminance of each dot was $12.8 \mathrm{~cd} / \mathrm{m}^{2}$; the luminance of the dark background was less than $0.4 \mathrm{~cd} / \mathrm{m}^{2}$.

Each experimental trial was composed of $2,3,4,5,6,8$, or 10 identical four-frame display cycles. A display cycle was formed as follows: Two dots corresponding to the opposite diagonal corners of an imaginary rectangle were presented for $195 \mathrm{msec}$, followed by a blank frame for $45 \mathrm{msec}$, followed by a frame in which two dots corresponding to the other diagonal corners of the imaginary rectangle were presented for $195 \mathrm{msec}$, followed again by a $45-\mathrm{msec}$ blank frame. The duration of each trial was quantified in terms of the number of display cycles.

The horizontal distance between dot positions remained constant at 4 pixels $\left(6.4^{\prime} ; 0.107^{\circ}\right)$ for all trials. The vertical distance remained constant within a trial but varied over trials; it was $3,4,5$, or 6 pixels, resulting in rectangular configurations with aspect ratios of $0.75,1.0,1.25$, or 1.5 . In order to minimize possible inter- trial effects, a seven-cycle "interrupting" stimulus was presented at the end of each trial (its effectiveness was not evaluated). The upper-left and lower-right dots of the motion quartet with an aspect ratio of 1.0 were presented in alternation (again for $195 \mathrm{msec}$ with intervening 45-msec blank frames), resulting in the perception of diagonal motion.

Design. Each testing session consisted of two sets of 224 trials. Each set was composed of eight blocks of 28 randomly ordered trials, the latter determined by the orthogonal combination of four aspect ratios of the motion quartet $(0.75,1.0,1.25$, or 1.5$)$ and seven trial durations $(2,3,4,5,6,8$, or 10 display cycles). Subjects J.G. and B.B. completed 12 experimental sessions; Subject A.V. completed 18 experimental sessions.

Procedure. Two responses were required at the conclusion of each trial. Subjects first responded to the initially perceived motion pattern (horizontal or vertical) by pressing one of two keys on the computer keyboard with fingers of their left hand. Then they pressed one of two keys with fingers of their right hand to indicate whether or not the perceived motion pattern had changed anytime during the trial.

\section{Results}

The data for each subject were sorted into eight subsets on the basis of the aspect ratio of the motion quartet $(0.75$, $1.0,1.25$, and 1.5 ), and whether the initially perceived motion was horizontal or vertical. As can be seen in Figure $2 \mathrm{a}$, both vertical and horizontal motion were seen for all four aspect ratios. For all 3 subjects, increases in the aspect ratio of the motion quartet resulted in the decreased perception of vertical motion and the increased perception of horizontal motion (as, e.g., in Hock et al., 1993).

\section{a) Initially Perceived Motion Pattern}
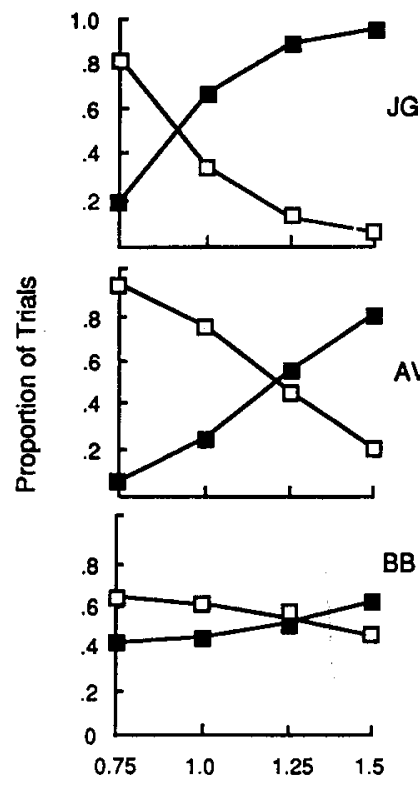

Aspect Ratio b) Change in the Perceived Motion Pattern
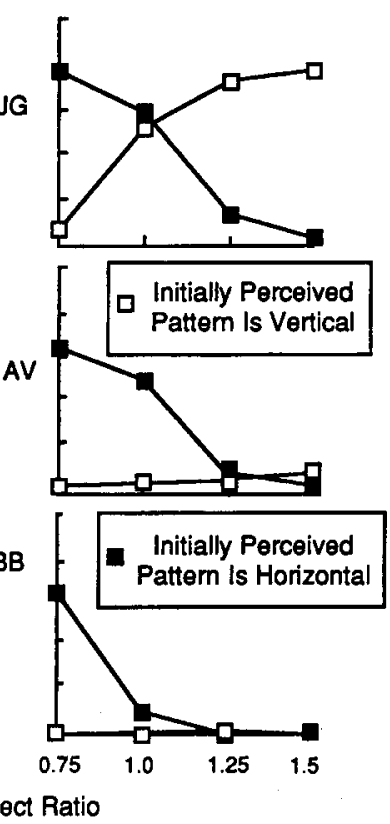

Figure 2. Experiment 1: Proportions of trials for which the initially perceived motion was either horizontal or vertical (a) and the proportion of perceived switches from horizontal to vertical motion and from vertical to horizontal (b) for each aspect ratio (the vertical divided by the horizontal distance between dot positions). 
Figure $2 \mathrm{~b}$ shows the proportion of trials with perceptual switches to vertical motion from an initial state of horizontal motion, and perceptual switches to horizontal motion from an initial state of vertical motion. The proportions of trials with switches are averaged over trials of varying duration because estimates of switching probability are based on very few points when the initial percept occurs infrequently; for example, for J.G., when the aspect ratio was 1.5 and there were three display cycles per trial, the initially perceived motion was vertical for only 11 trials spread over 24 sets of trials (two sets of trials in each of 12 testing sessions). It can be seen that the probability of spontaneous switches out of the initially perceived motion pattern was inversely related to the probability of the initial pattern. When horizontal motion was perceived for small aspect ratios (when it was not favored), switches to vertical motion were more frequent than when it was perceived for larger aspect ratios (when it was favored). An inverse relationship between whether a pattern is favored by the aspect ratio and its stability was also obtained when the initial motion was vertical, but it was observable only for J.G. (there were very few spontaneous switches from vertical to horizontal motion for A.V. and B.B.).

The fact that there is some passage of time prior to the occurrence of a spontaneous perceptual change does not necessarily mean that the probability of a switch per unit of time has increased as a function of time. Some passage of time also would be required to "capture" the relatively infrequent stochastic fluctuations that are large enough to produce a spontaneous perceptual change. The satiation hypothesis, which requires increases in the probability of pattern change as a function of time, was tested against the assumption of temporal independence, which specifies that the probability of a spontaneous pattern change remains constant as a function of time.

Switching functions (the probability of at least one spontaneous pattern change as a function of trial duration) were calculated on the basis of the assumption of temporal independence (i.e., the probability of a pattern change remains constant for each repetition of a display cycle). The probability of at least one switch anytime within a trial with $N$ display cycles is given by Equation 1 (derived in Appendix A), where $p_{1}$ represents the probability of the first switch.

Probability $[1$ or more switches $]=1-\left(1-p_{1}\right)^{N-1}$.

Calculated switching functions for a selected range of possible $p_{1}$ values are presented in Figure 3 (the broken lines). In general, the longer the trial duration (i.e., the greater the number of display cycles), the greater the likelihood of at least one switch since there are more opportunities for the switch to occur. Likewise, the greater the value of $p_{1}$ (the probability of a switch during a single display cycle), the greater the probability of there being at least one switch over a series of display cycles. For the assumption of temporal independence to be met, an empirically obtained switching function must follow one of a) Horizontal-to-Vertical Pattern Changes (first switch)

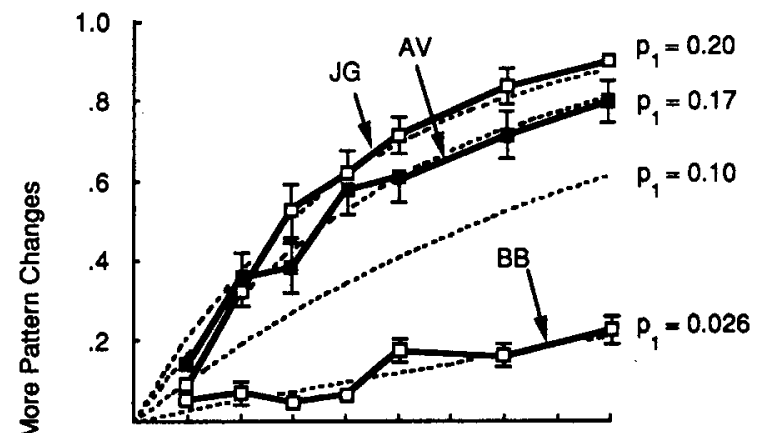

b) Vertical-to-Horizontal Pattern Changes (first switch)

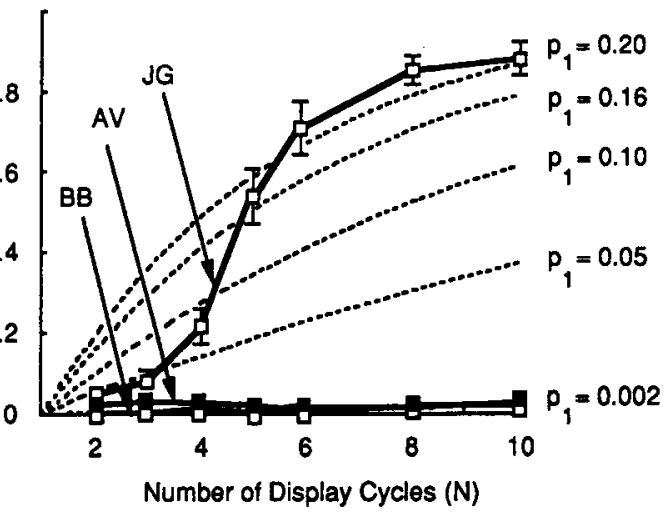

Figure 3. Experiment 1: Proportions of perceived switches from horizontal to vertical motion (a) and from vertical to horizontal motion (b) are indicated by bold lines (aspect ratio $=1.0$ only). Calculated temporal-independence functions are indicated by broken lines. Standard errors of the mean for each subject are indicated by error bars (when they are larger than the size of the symbols).

the calculated functions. This would show that the probability of a switch per unit of time (i.e., per display cycle) remained constant as a function of time elapsed since the start of the trial. Evidence for an influence of differential adaptation would be obtained if empirical switching functions, when compared to the calculated functions, indicate an increase in the probability of a switch as a function of trial duration.

The solid lines in Figure 3 show the empirically obtained proportions of trials for which subjects reported that there was at least one change in the perceived pattern. These data were obtained for trials in which the aspect ratio of the motion quartet was 1.0 , since only for this aspect ratio was there a sufficient number of switches to accurately estimate empirical switching functions. Comparing the empirical functions for horizontal-to-vertical switches with the calculated temporal-independence functions (Figure $3 a$ ) indicates for all 3 subjects that the probability $\left(p_{1}\right)$ of a switch during a display cycle remained constant for up to 10 display cycles (a duration of approximately $5 \mathrm{sec}$ ), consistent with the assumption 
of temporal independence. This evidence for temporal independence was obtained despite $p_{1}$ varying by an order of magnitude across the 3 subjects. (The brevity of the trials with only two display cycles made it difficult for subjects to both identify the initial motion pattern and determine whether it changed. This would account for the dip in J.G.'s and A.V.'s switching functions for trials with two display cycles.)

The data for vertical-to-horizontal switches for J.G. (Figure 3b) did not initially follow one of the calculated switching functions; her empirical switching function has a steeper slope than the calculated functions for trials with up to six display cycles, indicating that the probability of a switch (per display cycle) increased as a function of time. This increase then "leveled off" and followed the calculated, temporal-independence function representing a $p_{1}$ value of approximately 0.2 . Subjects A.V. and B.B. had too few changes from the perception of vertical to horizontal motion to measure their switching functions. This floor effect, which was the result of the very high stability of their vertical motion, hid any possible effect of trial duration on their vertical-to-horizontal switching probabilities.

\section{Discussion}

The primary purpose of Experiment 1 was to determine the time course of the first spontaneous pattern change following the onset of a bistable stimulus. The temporal independence observed for horizontal-to-vertical switches was consistent with what would be expected when a percept is relatively unstable. Low stability for horizontal motion implies that the activational advantage of horizontal over vertical motion detectors is small, so relatively small stochastic fluctuations are sufficient to reverse their activation and cause a spontaneous pattern change. Assuming that the amount of adaptation for a detector depends on its level of activation, adaptation-induced reductions in activation would be similar for perceived (horizontal) and unperceived (vertical) motion directions, and horizontal detectors would retain their small activational advantage. As a result, the likelihood of a stochastic fluctuation reversing the relative activational advantage of horizontal compared with vertical motion detectors would remain relatively constant as a function of time.

The temporal dependence observed for vertical-tohorizontal switches indicated that adaptation (satiation) can influence the rate of spontaneous pattern change. This was consistent with what would be expected when a percept is relatively stable. High stability for vertical motion implies that vertical motion detectors are much more activated than are horizontal motion detectors, so that stochastic fluctuations large enough to reverse their relative activation and cause a spontaneous loss of stability (pattern change) are rare. Again assuming that the amount of adaptation for a detector depends on its level of activation, adaptation-induced reductions in activation would be greater for perceived (vertical) than unperceived (horizontal) motion directions. This, however, does not mean that adaptation is the direct cause of the spontaneous vertical-to-horizontal switches. As timedependent adaptation reduces the initial activational advantage of the vertical motion detectors, the probability of a vertical-to-horizontal switch would increase because a smaller (more likely) stochastic fluctuation would be sufficient to reverse the relative activation of the detectors.

As indicated earlier, the central assumption of the satiation hypothesis is that there would be differential adaptation of the perceived and unperceived motion directions. Nondifferential adaptation, which would result in temporally independent spontaneous switching, can take the form of either equal adaptation of horizontal and vertical motion for the motion quartets or no adaptation of either. However, the evidence for an influence of adaptation on vertical-to-horizontal switches indicates that similar adaptation is more likely than no adaptation. In either case, it could be concluded that spontaneous switching from horizontal to vertical motion was observed that was not attributable to differential changes in sensitivity for detectors responsive to features of the perceived versus the unperceived pattern. In the absence of differential adaptation as the cause for the reversal in the relative activation of the detectors associated with the perceived and unperceived motion, the horizontal-to-vertical switches observed in Experiment 1 are attributable to stochastic fluctuations in detector activation (DeMarco et al., 1977; Hock et al., 1993; Sadler \& Mefferd, 1970; Taylor \& Aldridge, 1974).

Although the preceding account follows Hock et al. (1996) in emphasizing the dependence of adaptation effects on a percept's stability, the mechanisms responsible for the formation of stable perceptual patterns are as yet not fully understood. One likely contributor to the relative stability of the patterns perceived for the motion quartet is mutual inhibition between horizontal and vertical motion detectors. Evidence for such inhibitory competition has been reported by Snowden $(1989,1990)$ for random cinematograms and by Hock et al. for motion quartets. Differences in the strength and the symmetry of this mutual inhibition might have been responsible for vertical motion being so stable for A.V. and B.B.

The greater initial stability of vertical compared with horizontal motion for the 1.0 aspect ratio may also be related to whether or not the motion percept is established in the same or different cerebral hemispheres. For centrally fixated quartets, vertical motion is established within the same hemisphere, whereas horizontal motion is established across the two hemispheres. Gengerelli (1948) concluded that this was the basis for the small bias toward seeing vertical motion for the 1.0 aspect ratio (the vertical-horizontal illusion may also have been a factor), and it could also have contributed to the greater stability of vertical motion, especially for Subjects A.V. and B.B.

\section{EXPERIMENT 2}

As discussed above, if it is assumed that the amount of adaptation for a detector is proportional to its momentto-moment level of activation, then differential adapta- 
tion occurring for detectors associated with the perceived and unperceived motion would bring their respective activation levels closer together. This monotonic convergence would not result in the reversal of the relative activation of competing motion detectors (as required by the satiation hypothesis). However, it would be sufficient to account for the increased probability of vertical-tohorizontal switches as a function of time because the convergence of activation levels would result in smaller, more likely stochastic fluctuations being sufficient to reverse the relative activation of competing detectors. When adaptation effects asymptote (after about six display cycles for J.G. when the initial percept was vertical), switches depend only on stochastic fluctuations, and temporal independence is observed for vertical-to-horizontal as well as horizontal-to-vertical switches.

Convergence of activation levels might also account for the increased probability of successive pattern switches (Babich \& Standing, 1981; Cohen, 1959). That is, any stochastic fluctuation that is sufficient to reverse the relative activation of vertical and horizontal detectors would produce a perceptual switch that will tend, on the average, to leave the newly perceived pattern with a relatively small activational advantage over the unperceived pattern. A smaller, more likely stochastic fluctuation would then be sufficient to induce the next switch. This would stand in contrast with previous explanations that attribute the increasing switching probability of successive switches to incomplete recovery of the unperceived motion from earlier adaptation.

Our strategy in this experiment was to replicate earlier evidence for increases in switching probability following an earlier switch. However, the experiment was designed in such a way that the replication would occur under conditions that excluded earlier explanations for this effect. That is, the objective was to show that increases in switching probability following an earlier switch are not caused by the perceived motion adapting while the unperceived motion is only partially recovering from its earlier adaptation. In one condition, subjects judged whether or not there was at least one change in the perceived motion pattern anytime during a trial. In the second condition, subjects judged whether or not there were at least two changes in the perceived motion pattern anytime during a trial. Specifically, we studied the effect of a preceding horizontal-to-vertical switch on the probability of a vertical-to-horizontal switch $\left(p_{2 . v h}\right)$. It was shown in Experiment 1, for motion quartets with an aspect ratio of 1.0, that the first spontaneous horizontal-to-vertical switch during a trial is not based on the differential adaptation of horizontal and vertical motion detectors. Thus, if the likelihood of a vertical-to-horizontal switch is increased by a preceding horizontal-to-vertical switch, it would not be due to vertical motion detectors adapting while horizontal motion detectors are recovering from adaptation. That is, if there is recovery from adaptation, it would be concurrent for horizontal and vertical detectors because they adapt concurrently. As indicated above, the increased switch rate would instead be attributable to a smaller (more likely) stochastic fluctuation being sufficient to produce the second compared with the first spontaneous switch.

\section{Method}

The two possible sequences of perceived motion patterns for the motion quartets are indicated below $(\mathrm{V}=$ vertical motion pattern; $\mathrm{H}=$ horizontal motion pattern; the subscripts indicate the first, second, and third occurrence of each pattern in a sequence):

$$
\begin{aligned}
& \text { Sequence A: }\left(V_{1} \rightarrow H_{1}\right) \rightarrow V_{2} \rightarrow H_{2} \rightarrow V_{3} \rightarrow H_{3} \rightarrow \ldots \\
& \text { Sequence B: } H_{1} \rightarrow\left(V_{1} \rightarrow H_{2}\right) \rightarrow V_{2} \rightarrow H_{3} \rightarrow V_{3} \rightarrow \ldots
\end{aligned}
$$

Our analysis, which was based on the first two spontaneous pattern changes, compared probabilities of pattern change across Sequences $\mathrm{A}$ and $\mathrm{B}$. Thus, the probability per unit of time (i.e., per display cycle) of a vertical-to-horizontal switch was compared when it was the first switch following the onset of a trial ( $p_{1 . v h}$ in Sequence A) with when it was the second switch during a trial $\left(p_{2 . v h}\right.$ in Sequence $B$ ). That is, we determined whether the probability of a vertical-to-horizontal switch was unaffected by the occurrence of a preceding horizontal-to-vertical switch $\left(p_{2 . \mathrm{vh}}=p_{1 . \mathrm{vh}}\right)$, or whether the probability of a vertical-to-horizontal switch increased following the occurrence of a preceding horizontal-to-vertical switch $\left(p_{2 . \mathrm{vh}}>p_{1 . \mathrm{yh}}\right)$.

Subjects. Three well-practiced subjects, graduate students at Florida Atlantic University, participated in Experiment 2, two of whom (J.G. and A.V.) were also subjects in Experiment 1.

Stimuli. For Subjects J.G. and K.E., the stimuli were identical to those in Experiment 1 with the exception that only the 1.0 aspect ratio was tested. The number of display cycles in each trial was again $2,3,4,5,6,8$, or 10 , and a seven-cycle "interrupting" stimulus ended each frame with a diagonal motion pattern to minimize intertrial effects. The number of display cycle repetitions was substantially increased to accommodate Subject A.V., whose probability of spontaneous vertical-to-horizontal switches in Experiment 1 was very low. There were $8,11,14,17,20,25,30,35$, or 40 display cycles per trial, and the duration of the blank frames in each display cycle was reduced from 45 to $0 \mathrm{msec}$.

Design. There were 12 testing sessions, each with two sets of 224 trials. Each set consisted of eight randomized blocks of 28 trials (seven trial durations repeated four times). During one set of trials, subjects reported (after each trial) whether the initially perceived motion pattern was horizontal or vertical, and then whether or not there was at least one change in the perceived motion pattern anytime during the trial (as in Experiment 1). During the second set of trials, subjects reported whether the initially perceived motion pattern was horizontal or vertical, and then whether or not there were at least two changes in the perceived motion pattern anytime during a trial.

\section{Results: Subjects J.G. and K.E.}

"One or more" switches per trial. The results for this experiment were consistent with those of Experiment 1, even though only the 1.0 aspect ratio was tested (in Experiment 1 , the 1.0 aspect ratio was tested in the context of three other aspect ratios - $0.75,1.25$, and 1.5). In Figure $4 a$, horizontal-to-vertical switches (the initially perceived motion was horizontal) again followed calculated temporal-independence switching functions $\left(p_{1 . h v}=\right.$ constant) for both subjects (although over time there was a small increase in the probability of a switch for K.E.). A least squares procedure was used to find the temporal- 


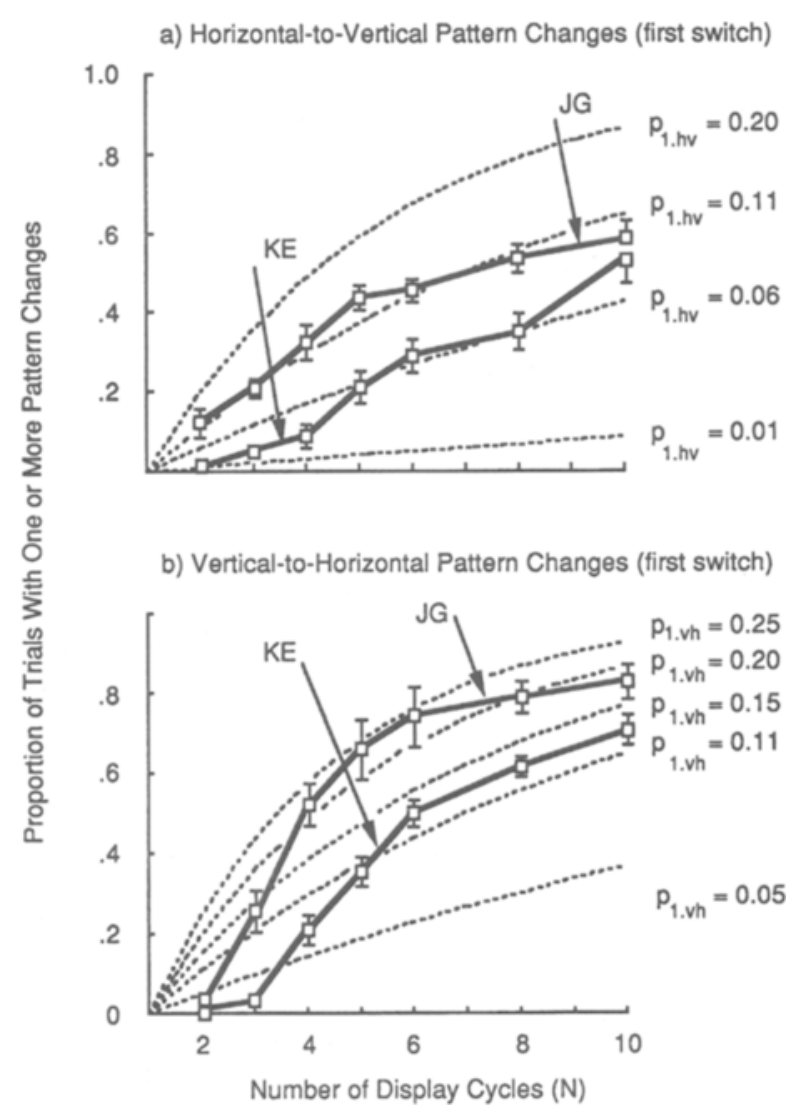

Figure 4. Experiment 2 (Subjects J.G. and K.E.): Proportions of perceived switches from horizontal to vertical motion (a) and from vertical to horizontal motion (b) are indicated by bold lines (aspect ratio $=1.0$ ). Calculated temporal-independence functions are indicated by broken lines. Standard errors of the mean for each subject are indicated by error bars (when they are larger than the size of the symbols).

independence switching functions that provided the best fit to each subject's empirical "one or more" switching function. The resulting switching probability during each display cycle was 0.11 for J.G. and 0.06 for K.E. As shown in Figure 4b, vertical-to-horizontal switches when the initially perceived motion was vertical $\left(p_{1 . v h}\right)$ again increased in probability over the first six display cycles.

"Two or more" switches per trial. Our objective in analyzing these data was to determine whether the probability of a vertical-to-horizontal switch was unaffected by the prior occurrence of a horizontal-to-vertical switch $\left(p_{2 . \mathrm{vh}}=p_{1 . \mathrm{vh}}\right)$, or whether its probability increased following the occurrence of a prior horizontal-to-vertical switch $\left(p_{2 . v \mathrm{vh}}>p_{1 . \mathrm{vh}}\right)$. The calculated switching functions for "two or more" pattern changes when the initially perceived motion was horizontal were obtained by determining probability values from the "one or more" data $\left(p_{1 . \mathrm{hv}}\right)$ and selecting a range of possible values for $p_{2 . v h}$, the probability of the second switch. The probability of two or more switches is indicated by Equation 2 (derived in Appen$\operatorname{dix} \mathrm{B}$ ), where $N$ is the number of display cycles per trial. These are once again temporal-independence switching functions in that they are based on $p_{1 \text { hv }}$ remaining constant following stimulus onset and $p_{2 \text { vh }}$ remaining constant following the first pattern change:

$$
\begin{aligned}
& \text { Probability [2 or more switches] }= \\
& \qquad\left[1-\left(1-p_{1, \mathrm{hv}}\right)^{N-1}\right]-\sum_{i=2}^{N}\left[p_{1 . \mathrm{hv}}\left(1-p_{1 . \mathrm{hv}}\right)^{N-i}\left(1-p_{2 . \mathrm{vh}}\right)^{i-2}\right] .
\end{aligned}
$$

The broken lines in Figure 5 represent values for the probability of two or more switches in a trial with $N$ display cycles that are calculated for Equation 2 on the basis of each subject's empirically determined value of $p_{1 . \mathrm{hv}}$ (least squares estimated values of 0.11 for J.G. and 0.06 for K.E.; Figure 4a), and a selected range of possible values for $p_{2 . v \mathrm{vh}}$. Since our analysis was concerned with whether or not $p_{2 . v \mathrm{v}}$ was greater than $p_{1 . \mathrm{vh}}$, some of the $p_{2 . v \mathrm{w}}$ values selected for the calculation of the probability of two or more switches were derived from the empirical

\section{a) Vertical-to-Horizontal Pattern Changes (second switch)}

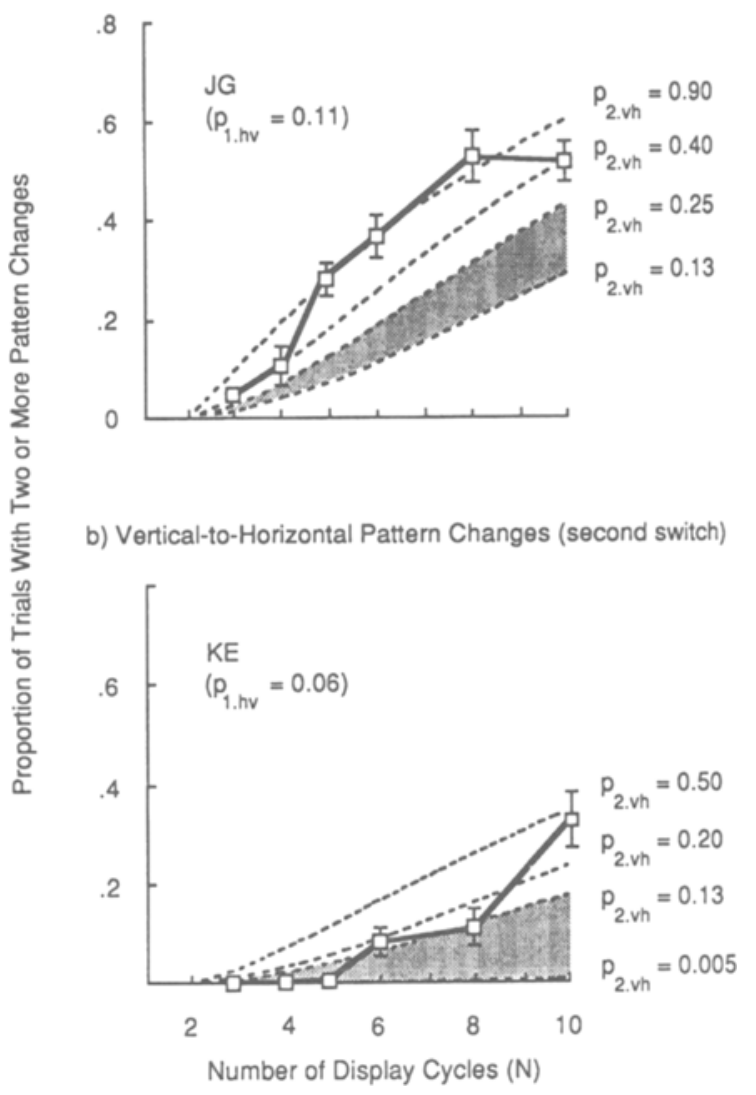

Figure 5. Experiment 2 (Subjects J.G. and K.E.): Proportions of two or more switches when the initially perceived motion was horizontal are indicated by bold lines. The gray areas in each graph represent the regions for which the probability of a verticalto-horizontal switch does not depend on whether or not there was a preceding pattern change (where $p_{1 . v h}=p_{2 . v h}$ ). Standard errors of the mean for each subject are indicated by error bars (when they are larger than the size of the symbols). 
"one or more" vertical-to-horizontal switches in Figure $4 \mathrm{~b}$. Starting with the results for three display cycles (the minimum trial duration for the occurrence of two switches), it can be seen in Figure $4 \mathrm{~b}$ that the value of $p_{1, v h}$ varies as a function of the number of display cycles (unlike the horizontal-to-vertical switches, the empirical vertical-to-horizontal switching functions do not come close to fitting calculated temporal-independence functions, so the least squares estimation of a single switching probability was not appropriate). Two of the selected values for J.G. $(0.13$ and 0.25$)$ and two of the selected values for K.E. (0.05 and 0.13$)$ corresponded to their lowest and highest values of $p_{1 . \mathrm{vh}}$ (Figure $4 \mathrm{~b}$ ). The probability ( 2 or more switches) calculated on the basis of these values defined the gray regions for J.G. and K.E. in Figure 5.

If the empirical "two or more" switching function was always within the gray region, the results would be consistent with $p_{2, v h}=p_{1 . v \mathrm{~h}}$. This, however, was not the case. As can be seen in Figure 5, the empirical "two or more" switching function rose above the gray region for both J.G. and K.E. This indicated that the probability of the vertical-to-horizontal switch was greater when it was the second than when it is the first switch (i.e., $p_{2 . \mathrm{vh}}>p_{1 . v \mathrm{~h}}$ ). The difference emerged most strongly for the longer trials because the second switch obviously could not occur until after the first and because the probability of spontaneous vertical-to-horizontal switches tends to increase as a function of time. The effect of a prior switch on the probability of a horizontal-to-vertical switch $\left(p_{2 . \text { hv }}\right)$ could not be evaluated in this experiment because the probability of the preceding vertical-to-horizontal switch $\left(p_{1 . v \mathrm{v}}\right)$ was not temporally independent (as required by the derivation in Appendix B).
Results: Subject A.V.

"One or more" switches per trial. Horizontal-tovertical switches again followed a calculated switching function ("one or more" responses, Figure 6). The very high stability of vertical motion for A.V. in Experiment 1 suggested that trials of longer duration would be necessary to capture the very infrequent stochastic fluctuations that would be large enough to produce a spontaneous switch to horizontal motion. Indeed, the fourfold increase in trial duration for A.V. in this experiment was successful in capturing these fluctuations, and the vertical-to-horizontal switches that were observed approximated a calculated switching function; that is, the switches were attributable to stochastic fluctuations rather than the differential adaptation of vertical and horizontal motion detectors. As above, a least squares procedure was used to find the temporal-independence switching functions that provided the best fit to A.V.'s empirical "one or more" switching functions. The resulting switching probability during each display cycle was 0.04 for her horizontal-to-vertical switches and 0.012 for her vertical-to-horizontal switches.

For J.G. in Experiments 1 and 2 and K.E. in Experiment 2 , increases in the probability of vertical-tohorizontal switches were restricted to the first six display cycles $(3 \mathrm{sec})$, then followed a calculated temporalindependence function. The briefest trial for A.V. in Experiment 2 was composed of eight display cycles, so the observation of temporal independence for her vertical/ horizontal switches was consistent with effects of differential adaptation occurring within about $3 \mathrm{sec}$ for the motion quartet.

"Two or more" switches per trial. The switching functions for A.V.'s "two or more" responses when the initial

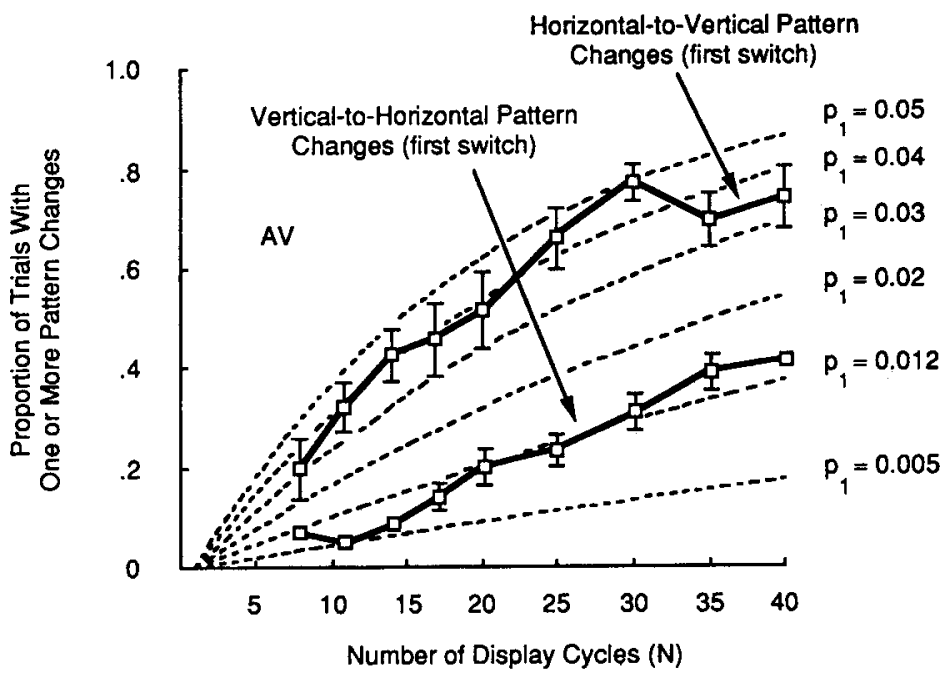

Figure 6. Experiment 2 (Subject A.V.): Proportions of perceived switches from horizontal to vertical motion and from vertical to horizontal motion are indicated by bold lines (aspect ratio $=1.0$ ). Calculated temporal-independence functions are indicated by broken lines. Standard errors of the mean for each subject are indicated by error bars (when they are larger than the size of the symbols). 
motion was horizontal were calculated from Equation 2 on the basis of the empirically determined best fit value of $p_{1 . h v}$ from her "one or more" responses and a selected set of possible values of $p_{2 . v h}$ (indicated by broken lines in Figure 7a). The gray area again represents the region associated with the assumption that $p_{2 . v h}=p_{1 . v h}$, and the empirical "two or more" switching function presented as a solid line again indicates that the probability of a spontaneous vertical-to-horizontal change was greater when it was the second than when it was the first pattern change during a trial $\left(p_{2 . \mathrm{vh}}>p_{1 . \mathrm{vh}}\right)$.

The effect of a prior switch on the probability of a horizontal-to-vertical switch ( $p_{2 . \mathrm{hv}}$ ) could also be evaluated for A.V. because the probability of the preceding vertical-to-horizontal switch $\left(p_{1 . v \mathrm{~h}}\right)$ was approximately temporally independent over the trial durations that were tested (as required by the derivation in Appendix B). The results were consistent with those obtained for verticalto-horizontal switches (Figure $7 \mathrm{~b}$ ). That is, the probability of a spontaneous horizontal-to-vertical change was greater when it was the second than when it was the first switch during a trial $\left(p_{2 . \mathrm{hv}}>p_{1 . \mathrm{hv}}\right)$.

\section{Discussion}

The results for reports of "two or more" pattern changes were consistent with previous evidence (e.g., Babich \& Standing, 1981; Cohen, 1959) that the likelihood of a pattern change increases with the number of preceding pattern changes. However, the increase in the probability of a vertical-to-horizontal switch $\left(p_{2 . \mathrm{vh}}>p_{1 . \mathrm{vh}}\right)$ cannot be accounted for by horizontal motion detectors recovering from adaptation while vertical motion detectors are adapting (and vice versa for A.V.'s horizontal-to-vertical switches). As indicated earlier, if there was recovery from adaptation, it would have been concurrent for horizontal and vertical detectors because if they adapted, they did so concurrently. This was indicated by the temporal independence of the first horizontal-to-vertical switch for J.G. and K.E., and the first horizontal-to-vertical and vertical-to-horizontal switches for A.V.

a) Vertical-to-Horizontal Pattern Changes (second switch)

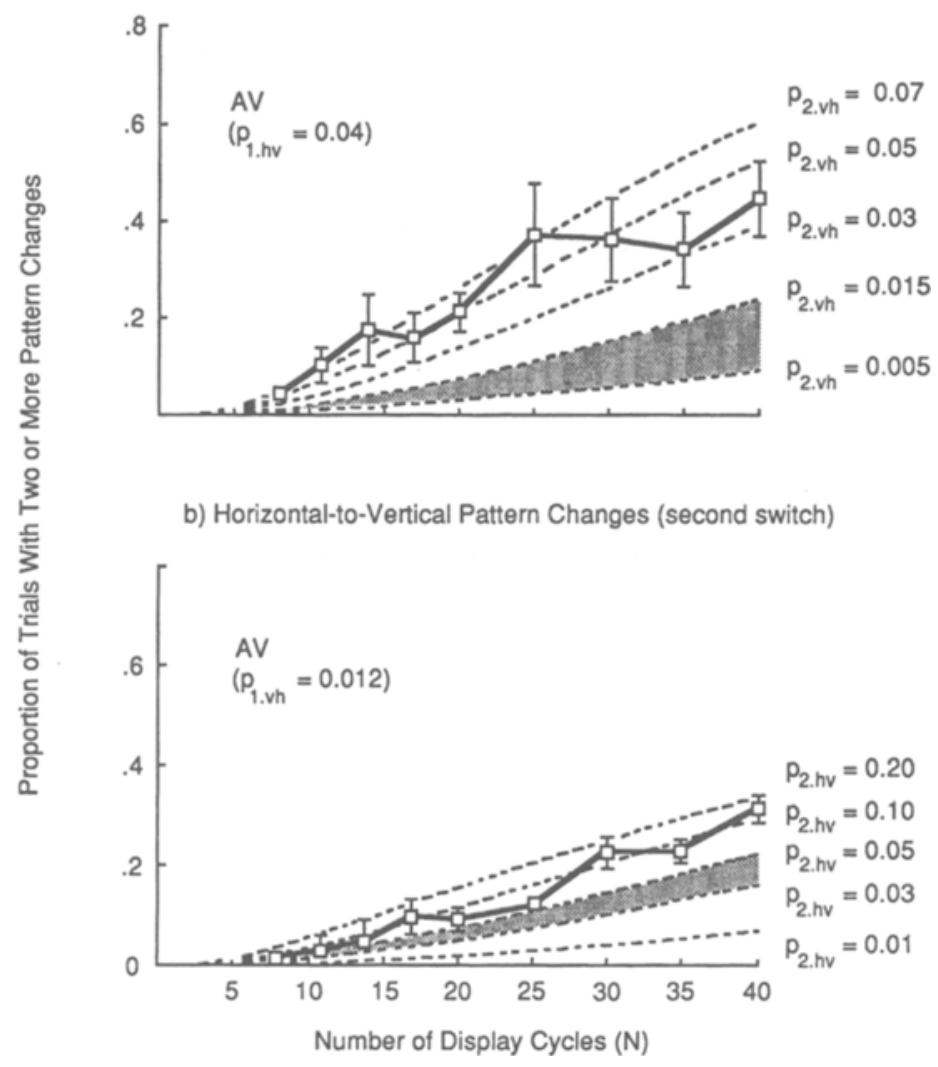

Figure 7. Experiment 2 (Subject A.V.): Proportions (and standard errors) of two or more switches when the initially perceived motion was horizontal (a) and when the initially perceived motion was vertical (b) are indicated by bold lines. The gray areas in each graph represent the regions for which a preceding pattern change did not affect the probability of vertical-to-horizontal switches (where $p_{1 . v h}=p_{2 . v h}$ for the upper graph) or horizontal-to-vertical switches (where $p_{1 . h v}=p_{2 . h v}$ for the lower graph). Standard errors of the mean for each subject are indicated by error bars (when they are larger than the size of the symbols). 
To summarize, the results for "one or more" switches (Experiments 1 and 2) indicated that differential adaptation (satiation) of the perceived and unperceived motion directions is not necessary to account for the first pattern change following the onset of a bistable stimulus. The results for "two or more" switches (Experiment 2) indicated that adaptation of the perceived motion accompanied by recovery from adaptation of the unperceived motion is not necessary to account for the increased probability of the second compared with the first switch.

\section{GENERAL DISCUSSION}

The idea that spontaneous changes between the patterns perceived for bistable stimuli occur because of timedependent adaptation of the perceived pattern is widely accepted. It has, for example, been the basis for the recently proposed models of Ditzinger and Haken (1989, 1990). Given the general prevalence of habituation and adaptation effects in a wide range of neural (see, e.g., Maffei, Fiorentini, \& Bisti, 1973) and perceptual (see, e.g., Walraven, Enroth-Cugell, Hood, MacLeod, \& Schnapf, 1990) phenomena, it would be expected that adaptation would influence the probability of spontaneous pattern change for a bistable stimulus. Nonetheless, it does not appear that reversals in relative activation resulting from the differential adaptation of perceived and unperceived pattern features are the direct cause of spontaneous pattern changes.

A number of investigators have shown that differential adaptation of the perceived and unperceived pattern is not sufficient for the occurrence of spontaneous pattern changes. Girgus, Rock, and Egatz (1977) and Rock and Mitchener (1992) have demonstrated that spontaneous changes from an initially established pattern are much less likely to occur when the subject is unaware of an alternative percept for the stimulus. Thus, the prolonged perception of one pattern for a bistable stimulus is not sufficient for it to adapt and be replaced by the unperceived (and presumably unadapted) alternative pattern. Intention can also influence the occurrence of spontaneous pattern changes. Peterson and Hochberg (1983) showed that an intentional effort to maintain a perceived pattern reduces the likelihood of it changing; its adaptation relative to that of the unperceived alternative pattern is not sufficient to produce a spontaneous pattern change.

In this article, we have provided evidence that the differential adaptation of the perceived and unperceived pattern is not necessary for spontaneous pattern changes. According to the satiation hypothesis, the likelihood of a spontaneous pattern change for a bistable stimulus should always increase as a function of the time elapsed since the formation of the perceived pattern (since only the latter is presumed to adapt). Taylor and Aldridge (1974) previously showed that this is not the case when the time between pattern changes was analyzed over a long series of spontaneous changes, and we have shown (for horizontal-to-vertical switches) that time-dependent differential adaptation of perceived and unperceived pattern features is not necessary for the occurrence of the first pattern change following the onset of a bistable stimulus. The increase in the probability of a pattern change as a function of the number of preceding pattern changes has also been cited as supportive of the satiation hypothesis (see, e.g., Cornwell, 1976; Spitz \& Lipman, 1962). We also have shown that the probability of a pattern change increases when it is preceded by another pattern change (at least for the first two pattern changes). However, we have done so when there was no preceding differential adaptation of the perceived and unperceived motion. Hence, the increased switching probability cannot be attributed to the perceived pattern adapting while the previously perceived pattern recovers from adaptation (reversing their relative activation).

If differential adaptation (satiation) of perceived and unperceived pattern features is neither necessary nor sufficient for spontaneous pattern change, does it at least influence the probability of a pattern change? The time dependence of vertical-to-horizontal switches observed in Experiments 1 and 2 indicates that differential adaptation can influence the likelihood of a pattern change when the adapting pattern is highly stable (see also Long et al., 1992). Assuming that the degree of adaptive reductions in detector sensitivity depends on the initial level of detector activation, the effect of adaptation would be to bring disparate levels of activation for horizontal and vertical detectors closer together, increasing the likelihood of a stochastic fluctuation reversing their relative activation.

A stochastically induced pattern change would tend to increase the probability of the next pattern change for the same reason. For example, stochastic fluctuations that overcome an initial advantage in activation for horizontal relative to vertical motion detectors will result, on average, in a relatively small activational advantage for the vertical detectors compared with when the vertical pattern is established upon the onset of the motion quartet (i.e., the switch will leave the activational state of the motion detectors close to the boundary separating the vertical and horizontal percepts). As a result, the occurrence of a spontaneous vertical-to-horizontal switch is more likely when there has been a prior horizontal-to-vertical switch - that is, when the required stochastic fluctuation is smaller (Experiment 2).

In conclusion, the results of this study indicate that adaptation and stochastic fluctuations are not mutually exclusive; alternative influences on spontaneous pattern change. Adaptation can influence the probability of spontaneous change by bringing activation levels of competing detectors closer together. However, the hypothesis that spontaneous pattern changes can occur only when adaptation (satiation) reverses the relative activation of competing detectors is not supported. Such reversals appear instead to be the result of stochastic fluctuations in their relative activation. 


\section{REFERENCES}

Babich, S., \& Standing, L. (1981). Satiation effects with reversible figures. Perceptual \& Motor Skills, 52, 203-210.

Blakemore, C., \& CAMPBELL, F. W. (1969). On the existence of neurones in the human visual system selectively sensitive to the orientation and size of retinal images. Journal of Physiology, 203, 237-260.

Carlson, V. R. (1953). Satiation in a reversible perspective figure. Journal of Experimental Psychology, 45, 442-448.

COHEN, L. (1959). Rate of apparent change of a Necker cube as a function of prior stimulation. American Journal of Psychology, 72, 327-344.

CoRNwell, H. G. (1976). Necker cube reversal: Sensory or psychological satiation? Perceptual \& Motor Skills, 43, 3-10.

DeMarco, A., Pennengo, P., Trabucco, A., Borsellino, A., CarLINI, F., Riani, M., \& Tuccio, M. T. (1977). Stochastic models and fluctuations in reversal time of ambiguous figures. Perception, 6 , 645-656.

DITZINGER, T., \& HAKEN, H. (1989). Oscillations in the perception of ambiguous patterns: A model based on synergetics. Biological Cybernetics, 61, 279-287.

Ditzinger, T., \& HAKEN, H. (1990). The impact of fluctuations on the recognition of ambiguous patterns. Biological Cybernetics, 63, 453-456.

GeNGERELLI, J. A. (1948). Apparent motion in relation to homonymous and heteronymous stimulation of the cerebral hemispheres. Journal of Experimental Psychology, 38, 592-599.

GiRGUS, J. J., Rock, I., \& EGATZ, R. (1977). The effect of knowledge of reversibility on the reversibility of ambiguous figures. Perception \& Psychophysics, 22, 550-556.

HE, Z. J., \& NAKAYAMA, K. (1994). Apparent motion determined by surface layout not by disparity or three-dimensional distance. Nature, 367, 173-175.

HOCHBERG, J. E. (1950). Figure-ground reversal as a function of visual satiation. Journal of Experimental Psychology, 40, 682-686.

Hock, H. S., Kelso, J. A. S., \& SchönER, G. (1993). Bistability and hysteresis in the organization of apparent motion patterns. Journal of Experimental Psychology: Human Perception \& Performance, 19, 63-80.

Hock, H. S., Schöner, G., \& Hochstein, S. (1996). Perceptual stability and the selective adaptation of perceived and unperceived motion directions. Vision Research, 36, 3311-3323.

HOETH, F. (1968). Bevorzugte Richtungen bei stroboskopischen Alternativbewegungen [Preferred directions in stroboscopic alternative motion]. Psychologische Beiträge, 16, 494-527.

JASTROW, J. (1900). Fact and fable in psychology. Boston: Houghton Mifflin.

KöHLER, W., \& Wallach, H. (1944). Figural after-effects: An investigation of visual processes. Proceedings of the American Philosophical Society, 88, 269-357.

Kruse, P., Stadler, M., \& Wehner, T. (1986). Direction and frequency specific processing in the perception of long-range apparent motion. Vision Research, 26, 327-335.
Long, G. M., Toppino, T. C., \& Mondin, G. W. (1992). Prime time: Fatigue and set effects in the perception of reversible figures. Perception \& Psychophysics, 52, 609-616.

MAFFEI, L., Fiorentini, A., \& Bisti, S. (1973). Neural correlate of perceptual adaptation to gratings. Science, 182, 1036-1038.

MCDougaLL, W. (1906). The physiological factors in the attention process. Mind, 15, 329-359.

Metzger, W. (1975). Gestaltheorie in der modernen Psychologie. Darmstadt: Steinkopff. (Original work published 1941)

NAWROT, M., \& BLAKE, R. (1989). Neural integration of information specifying structure from stereopsis and motion. Science, 244, 716-718.

Pantle, A. J., \& SeKuler, R. (1968). Size detecting mechanisms in human vision. Science, 162, 1146-1148.

Pantle, A. J., \& Sekuler, R. (1969). Contrast response of human visual mechanisms sensitive to orientation and direction of motion. $\mathrm{Vi}$ sion Research, 9, 397-406.

Peterson, M. A., \& Hochberg, J. (1983). Opposed-set measurement procedure: A quantitative analysis of the role of local cues and intention in form perception. Journal of Experimental Psychology: Human Perception \& Performance, 9, 183-193.

Ramachandran, V. S., \& AnStis, S. M. (1985). Perceptual organization in multistable apparent motion. Perception, 14, 135-143.

Rock, I., \& MITCHENER, K. (1992). Further evidence of failure of reversal of ambiguous figures by uninformed subjects. Perception, 21, 39-45.

SADLER, T. G., \& MeFFERD, R. B., JR. (1970). Fluctuations of perceptual organization and orientation: Stochastic (random) or steady state (satiation)? Perceptual \& Motor Skills, 31, 739-749.

SEXULER, R., \& GANZ, L. (1963). A new aftereffect of seen movement with a stabilized retinal image. Science, 139, 1146-1148.

SNOWDEN, R. J. (1989). Motions in orthogonal directions are mutually suppressive. Journal of the Optical Society of America A, 6, 1096-1 101.

SNOWDEN, R. J. (1990). Suppressive interactions between moving patterns: Role of velocity. Perception \& Psychophysics, 47, 74-78.

SPITZ, S., \& LiPMAN, L. (1962). Some factors affecting Necker cube reversal rate. Perceptual \& Motor Skills, 15, 611-625.

TAYLOR, M. M., \& ALDRIDGE, K. D. (1974). Stochastic processes in reversing figure perception. Perception \& Psychophysics, 16, 9-27.

THOMPSON, P. (1981). Velocity after-effects: The effects of adaptation to moving stimuli on the perception of subsequently seen moving stimuli. Vision Research, 21, 337-345.

TROXLER, D. (1804). Über das Verschwinden gegebener Gegenstände innerhalb unseres Gesichtskreises [On the disappearance of given objects in visual space]. In K. Himly \& J. A. Schmidt (Eds.), Ophthalmische Bibliothek (Vol. 2, pp. 51-53). Jena: Fromann.

VON SCHILLER, P. (1933). Stroboskopische Alternativversuche [Stroboscopic alternative motion]. Psychologische Forschung, 17, 179-214.

Walraven, J., Enroth-Cugell, C., Hood, D. C., MacLeod, D. I. A., \& SChNAPF, J. L. (1990). The control of visual sensitivity: Receptoral and postreceptoral processes. In L. Spillman \& J. S. Werner (Eds.), Visual perception: The neurophysiological foundations (pp. 53-101). New York: Academic Press. 
The probability of there being one or more switches during a trial is

Probability [ 1 or more switches] $=1$ - probability [no switches].

The probability of no switches is determined as follows.

Let $p_{1}=$ probability of the first switch $(H \rightarrow V$ or $H \rightarrow V), N=$ number of display cycles per trial.

When the initially perceived motion is horizontal,

$$
\mathrm{H} \stackrel{\left(1-p_{1}\right)}{\rightarrow} \mathrm{H} \stackrel{\left(1-p_{1}\right)}{\rightarrow} \mathrm{H} \stackrel{\left(1-p_{1}\right)}{\rightarrow} \mathrm{H} \stackrel{\left(1-p_{1}\right)}{\rightarrow} \mathrm{H} \ldots \stackrel{\left(1-p_{1}\right)}{\rightarrow} \mathrm{H},
$$

$\left(1-p_{1}\right)$ is the probability of there not being a switch during a display cycle.

Then,

$$
\text { Probability [no switches] }=\left(1-p_{1}\right)^{N-1}
$$

and

$$
\text { Probability }[1 \text { or more switches }]=1-\left(1-p_{1}\right)^{N-1} \text {. }
$$

Note- $\mathrm{H}$, horizontal; $\mathrm{V}$, vertical.

\section{APPENDIX B}

Let $p_{1 \mathrm{hv}}=$ probability of horizontal/vertical switch when it is the first switch, $p_{1 . \mathrm{vh}}=$ probability of vertical horizontal switch when it is the first switch, $p_{2 . \mathrm{hv}}=$ probability of horizontal/vertical switch when it is the second switch, $p_{2 . v \mathrm{~h}}=$ probability of vertical/horizontal switch when it is the second switch, $N=$ number of display cycles per trial.

The probability of there being two or more switches during a trial is

Probability [ 2 or more switches] $=$ Probability [1 or more switches] - probability [exactly 1 switch].

The probability of exactly one switch is determined inductively for the case in which the initially perceived motion is horizontal:

When $N=2$, there is only one sequence with exactly one switch:

$$
\mathrm{H} \stackrel{\left(p_{1, h v}\right)}{\rightarrow} \mathrm{V} \quad \text { Probability [exactly } 1 \text { switch] }=p_{1 . \mathrm{hv}} .
$$

When $N=3$, there are two sequences with exactly one switch:

$$
\begin{array}{ll}
\mathrm{H} \stackrel{\left(p_{\text {thv }}\right)}{\rightarrow} \mathrm{V} \stackrel{\left(1-p_{2 v h}\right)}{\rightarrow} \mathrm{V} & \text { (switch is on the second display cycle). } \\
\mathrm{H} \stackrel{\left(1-p_{\text {l.h. }}\right)}{\rightarrow} \mathrm{H} \stackrel{\left(p_{\text {lh }}\right)}{\rightarrow} \mathrm{V} & \text { (switch is on the third display cycle). }
\end{array}
$$

Summing,

$$
\text { Probability [exactly } 1 \text { switch }]=\left(p_{1 . \text { hv }}\right)\left(1-p_{2 . \mathrm{vh}}\right)+\left(1-p_{1 . \mathrm{hv}}\right)\left(p^{1 . h v}\right) .
$$

When $N=5$, there are four sequences with exactly one switch:

$$
\begin{aligned}
& \mathrm{H} \stackrel{\left(p_{1, h v}\right)}{\rightarrow} \mathrm{V} \stackrel{\left(1-p_{2, \mathrm{n}}\right)}{\rightarrow} \mathrm{V} \stackrel{\left(1-p_{2, \mathrm{nh}}\right)}{\rightarrow} \mathrm{V} \stackrel{\left(1-p_{2, \mathrm{n}}\right)}{\rightarrow} \mathrm{V} \quad \text { (switch is on second display cycle). } \\
& \mathrm{H} \stackrel{\left(1-p_{1, h v}\right)}{\rightarrow} \mathrm{H} \stackrel{\left(p_{1, \mathrm{hl}}\right)}{\rightarrow} \mathrm{V} \stackrel{\left(1-p_{2, \mathrm{~h}}\right)}{\rightarrow} \mathrm{V} \stackrel{\left(1-p_{2, \mathrm{~h}}\right)}{\rightarrow} \mathrm{V} \quad \text { (switch is on third display cycle). } \\
& \mathrm{H} \stackrel{\left(1-p_{\left.1, h_{2}\right)}\right.}{\rightarrow} \mathrm{H} \stackrel{\left(1-p_{1, h_{2}}\right)}{\rightarrow} \mathrm{H} \stackrel{\left(p_{1, h_{2}}\right)}{\rightarrow} \mathrm{V} \stackrel{\left(1-p_{2 . \mathrm{h}}\right)}{\rightarrow} \mathrm{V} \quad \text { (switch is on fourth display cycle). }
\end{aligned}
$$

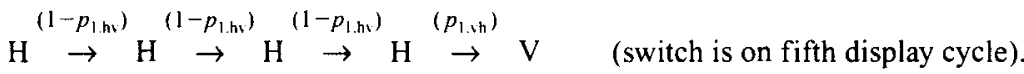


522

HOCK, SCHÖNER, AND VOLS

APPENDIX B (Continued)

Summing,

$$
\begin{aligned}
\text { Probability [exactly } 1 \text { switch] }= & \left(p_{1 . \mathrm{hv}}\right)\left(1-p_{2 . \mathrm{vh}}\right)\left(1-p_{2 . \mathrm{vh}}\right)\left(1-p_{2 . \mathrm{vh}}\right)+\left(1-p_{1 . \mathrm{hv}}\right)\left(p_{1 . \mathrm{hv}}\right)\left(1-p_{2 . \mathrm{vh}}\right)\left(1-p_{2 . \mathrm{vh}}\right) \\
& +\left(1-p_{1 . \mathrm{hv}}\right)\left(1-p_{1 . \mathrm{hv}}\right)\left(p_{1 . \mathrm{hv}}\right)\left(1-p_{2 . \mathrm{vh}}\right)+\left(1-p_{1 . \mathrm{hv}}\right)\left(1-p_{1 . \mathrm{hv}}\right)\left(1-p_{1 . \mathrm{hv}}\right)\left(p_{1 . \mathrm{vh}}\right) \\
\text { Probability [exactly 1 switch] }= & p_{1 . \mathrm{hv}}\left(1-p_{2 . \mathrm{vh}}\right)^{3}+p_{1 . \mathrm{hv}}\left(1-p_{1 . \mathrm{hv}}\right)\left(1-p_{2 . \mathrm{vh}}\right)^{2}+p_{1 . \mathrm{hv}}\left(1-p_{1 . \mathrm{hv}}\right)^{2}\left(1-p_{2 . \mathrm{vh}}\right) \\
& +p_{1 . \mathrm{hv}}\left(1-p_{1 . \mathrm{hv}}\right)^{3}
\end{aligned}
$$

Therefore, for any value of $N$, there are $N-1$ sequences with exactly one switch:

$$
\text { Probability [exactly } 1 \text { switch] }=\sum_{i=2}^{N}\left(p_{1 . \mathrm{hv}}\right)\left(1-p_{1 . \mathrm{hv}}\right)^{N-i}\left(1-p_{2 . \mathrm{vh}}\right)^{i-2} \text {. }
$$

Since,

Probability [ 2 or more switches] = Probability [1 or more switches] - Probability [exactly 1 switch].

From Appendix A (when the initial motion is horizontal),

$$
\text { Probability }[1 \text { or more switches }]=1-\left(1-p_{1 . \mathrm{hv}}\right)^{N-1} \text {. }
$$

Therefore,

Probability [2 or more switches] $=1-\left(1-p_{1 . \mathrm{hv}}\right)^{N-1}-\sum_{i=2}^{N}\left(p_{1 . \mathrm{hv}}\right)\left(1-p_{1 . \mathrm{hv}}\right)^{N-i}\left(1-p_{2 . \mathrm{vh}}\right)^{i-2}$

When the initially perceived motion is vertical,

Probability $[2$ or more switches $]=1-\left(1-p_{1 . \mathrm{vh}}\right)^{N-1}-\sum_{i=2}^{N}\left(p_{1 . \mathrm{vh}}\right)\left(1-p_{1 . \mathrm{vh}}\right)^{N-i}\left(1-p_{2 . \mathrm{hv}}\right)^{i-2}$.

(Manuscript received November 15, 1995;

revision accepted for publication May 29, 1996.) 